\title{
Predicting the future from the past: volatile markers for respiratory infections
}

\author{
To the Editor:
}

We read with great interest the editorial by AGUSTI [1] which reflected on past advances in technology in general, in respiratory medicine more specifically, and which tried to predict potential future advances. Among the latter, the use of exhaled biomarkers for detection of lung cancer caught our attention and we want to bring the editor's and readers' attention to a rapidly advancing related field of respiratory medicine, namely the analysis of volatile biomarkers of bacterial infections in exhaled breath. Like all living cells, bacteria produce numerous volatile organic compounds (VOCs), of which some may be species-specific and thus provide a biological fingerprint of pathogen presence. Detection of such bacteria-derived metabolites in exhaled breath is completely non-invasive and can be performed repeatedly without any burden to the patient. It is increasingly recognised as an innovative and promising diagnostic technique for early detection of emerging pulmonary infections [2]. Furthermore, breath analysis has high potential to advance precision diagnosis of pathogens as it reflects individual susceptibility to disease causing agents and also to therapeutic agents. Apart from this, the potential for non-invasive "real-time" analysis of exhaled biomarkers and the application of portable, point-of-care, sensor-based devices for personalised monitoring of disease state and therapy progress is a clear advancement beyond the current state-of-the-art in clinical practice. Ultimately such methodology could lead to more timely and hence more effective antibiotic use.

Clinical implementation as a standardised diagnostic tool will, however, require further research. To this end, numerous in vitro experiments have been carried out to identify pathogen-specific VOCs and to investigate factors influencing their production (including culture media and environmental conditions, initial bacterial load and growth phase, inter-strain variation, fungal-bacterial interactions and drug susceptibility). Species-specific VOCs were found and the kinetics of their production found to be related to pathogen load [3]. Recent studies have extended knowledge on underlying metabolic pathways and the differentiation between pathogen-derived metabolites and VOCs originating from the human microbiome, or host immune and non-immune cells. BEAN et al. [4] recently used a mouse model to correlate breath VOCs with the host's immune response and demonstrated that bacteria-induced changes in VOC levels are selective predictors for acute lung infections with Staphylococcus aureus and Pseudomonas aeruginosa. Similarly, KLEBER et al. [5] linked alterations in exhalation profiles for 3-pentanone, in the course of lipopolysaccharide (LPS)- and tumour necrosis factor (TNF)-induced inflammation in ventilated rats, to the metabolism of branched-chain keto acids governed by dynamically changing demand for substrates for cellular energy.

To further advance the establishment of a genotype-phenotype relationship for VOC production by pathogens, Bos et al. [6] demonstrated the ability of bacteria to produce acetaldehyde, ethanol and methanol by linking the results of metagenomic sequencing of bacterial DNA fragments in the sputum of cystic fibrosis (CF) patients with the Kyoto Encyclopedia of Genes and Genomes (KEGG) database. By combining these findings with the breath profiles of the CF patients who donated the sputum samples, the potential of breath-derived VOCs to predict respiratory tract colonisation/infection was illustrated. To further evaluate the metabolic activity of genetically encoded pathways of VOC production, PHAN et al. [7] added stable, isotope-labelled substrates $\left({ }^{13} \mathrm{C}_{6}\right.$-D-glucose and $\left.{ }^{2} \mathrm{H}_{2} \mathrm{O}\right)$ to cultures of Pseudomonas aeruginosa, Rothia mucilaginosa and Streptococcus salivarius. Analysis of the ${ }^{13} \mathrm{C}$-labelled products revealed that acetaldehyde and ethanol, for example, were not produced through the glycolytic pathway but instead through a process involving the hydrolysis of ${ }^{2} \mathrm{H}_{2} \mathrm{O}$. Further stable-isotope labelling experiments would help to confirm the microbial origin of a particular metabolite and may play a role in future investigation of the metabolic interactions occurring during polymicrobial infection.

Translation of this approach into the clinical setting was recently achieved by us in a prospective pilot clinical study where volatile metabolites produced by Staphylococcus aureus and Candida albicans under in vitro

@ERSpublications

Volatile organic compounds (VOCs) for the detection of respiratory infections http://ow.ly/rdhy30aEGQt

Cite this article as: Filipiak W, Ager C, Troppmair J. Predicting the future from the past: volatile markers for respiratory infections. Eur Respir J 2017; 49: 1700264 [https://doi.org/10.1183/13993003.00264-2017]. 
conditions were monitored in the breath of mechanically-ventilated patients. In persons infected with these microbes, the concentration profiles of exhaled VOCs corresponded to the severity of ventilation-associated pneumonia (VAP) [8]. This approach also appeared to work in patients where conventional cultivation of microorganisms from isolates failed, but where a change in the exhaled concentration profile of species-specific volatiles (e.g. 1-undecene) still hinted at the presence of a pathogen (most likely Pseudomonas aeruginosa) based on previously established in vitro profiles [8].

Considering the frequent lack of reproducibility associated with many findings [9], multicentre studies focused on promising biomarker candidates are an urgent next step. Here, key parameters of a future breath test (e.g. sensitivity and specificity) should be evaluated on independent cohorts of patients and healthy controls. Advancement in analytical techniques, especially in small and VOC-selective sensor arrays, should enable larger studies, improving their statistical power. Cheap, miniaturised "eNose" sensors have already been used in pilot clinical studies inter alia, to identify bacterial colonisation in clinically stable patients with chronic obstructive pulmonary disease [10], and they will ultimately lead to the personalised monitoring of disease state and therapy control.

Wojciech Filipiak ${ }^{1,2}$, Clemens Ager ${ }^{1,3}$ and Jakob Troppmair ${ }^{4}$

${ }^{1}$ Breath Research Institute, University of Innsbruck, Dornbirn, Austria. ${ }^{2}$ Dept of Pharmacodynamics and Molecular Pharmacology, Faculty of Pharmacy, Collegium Medicum in Bydgoszcz, Nicolaus Copernicus University in Torun, Torun, Poland. ${ }^{3}$ Dept of Anesthesiology and Critical Care Medicine, Innsbruck Medical University, Innsbruck, Austria. ${ }^{4}$ Daniel-Swarovski Research Laboratory, Dept of Visceral, Transplant and Thoracic Surgery, Innsbruck Medical University, Innsbruck, Austria.

Correspondence: Jakob Troppmair, Daniel-Swarovski Research Laboratory, Dept of Visceral, Transplant and Thoracic Surgery, Innsbruck Medical University, Innrain 66, 6020 Innsbruck, Austria. E-mail: jakob.troppmair@i-med.ac.at

Received: Feb 062017 | Accepted after revision: Feb 202017

Conflict of interest: None declared.

\section{References}

Agustí A. Predicting the future from the past. Eur Respir J 2017; 49: 1601854.

2 Macht M, Robinson JC, Graham BB. Updated approach for the assessment of ventilator-associated pneumonia. Crit Care Med 2013; 41: 2641-2642.

3 Filipiak W, Sponring A, Baur MM, et al. Molecular analysis of volatile metabolites released specifically by Staphylococcus aureus and Pseudomonas aeruginosa. BMC Microbiol 2012; 12: 113.

4 Bean HD, Jiménez-Díaz J, Zhu J, et al. Breathprints of model murine bacterial lung infections are linked with immune response. Eur Respir J 2015; 45: 181-190.

5 Kleber A, Maurer F, Lorenz D, et al. Metabolism of 3-pentanone under inflammatory conditions. J Breath Res 2016; 10: 047101.

6 Bos LD, Meinardi S, Blake D, et al. Bacteria in the airways of patients with cystic fibrosis are genetically capable of producing VOCs in breath. J Breath Res 2016; 10: 047103.

7 Phan J, Meinardi S, Barletta B, et al. Stable isotope profiles reveal active production of VOCs from human-associated microbes. J Breath Res 2017; 11: 017101.

8 Filipiak W, Beer R, Sponring A, et al. Breath analysis for in vivo detection of pathogens related to ventilator-associated pneumonia in intensive care patients: a prospective pilot study. J Breath Res 2015; 9: 016004

9 Baker M. Is there a reproducibility crisis? Nature 2016; 533: 452-454.

10 Sibila O, Garcia-Bellmunt L, Giner J, et al. Identification of airway bacterial colonization by an electronic nose in chronic obstructive pulmonary disease. Respir Med 2014; 108: 1608-1614.

Copyright @ERS 2017

From the author:

I thank Drs Filipiak, Ager and Troppmair for their interest in my editorial. This is exactly the type of discussion I was hoping to raise. Needless to say, I agree with their proposal.

Alvar Agusti ${ }^{1,2}$

${ }^{1}$ Respiratory Institute, Hospital Clínic, Institut d'Investigacions Biomèdiques August Pi i Sunyer (IDIBAPS), University of Barcelona, Barcelona, Spain. ${ }^{2}$ Centro de Investigación Biomédica en red Enfermedades Respiratorias (CIBERES), Madrid, Spain.

Correspondence: Alvar Agustí, Respiratory Institute, Hospital Clínic, Villarroel 170, 08036 Barcelona, Spain.

E-mail: AAGUSTI@clinic.cat

Received: March 022017 | Accepted: March 022017

Conflict of interest: Disclosures can be found alongside this article at erj.ersjournals.com 